\title{
BODY COMPOSITION AFTER TREATMENT OF SUBCLINICAL HYPERTHYRODISM
}

Authors Diana Boj-Carceller ${ }^{1}$, Alejandro Sanz-París ${ }^{1}$, Enrique Sánchez-Oriz², Rafael García-Foncillas López², Pilar CalmarzaCalmarza ${ }^{3}$, Vicente Blay-Cortes ${ }^{4}, \mathrm{M}^{\mathrm{a}}$ Dolores Abós-Olivares ${ }^{5}$

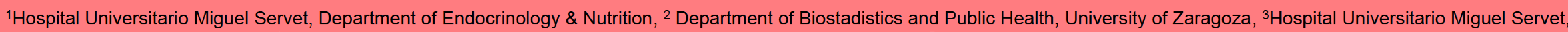
Service of Clinical Biochemistry, ${ }^{4}$ Hospital General de la Defensa Orad y Gajías, Department of Endocrinology, ${ }^{5}$ Hospital Universitario Miguel Servet, Department of Nuclear Medicine

\section{Background: Subclinical Hyperthyroidism (SHT) is associated with harmful effects on cardiovascular system, bone metabolism and progression to clinical hyperthyroidism. Loss of weight is a common fact in patients with clinical hyperthyroidism and of particular relevance in elderly patients, who are vulnerable to sarcopenia.}
Objective:
To assess changes in body composition after radioiodine therapy for SHT due to toxic nodular goiter.

\section{Methods:}

Prospective controlled cohort study. Patients with persistent SHT due to toxic nodular goiter received a single fixed dose of ${ }^{131}$ ( $(555 \mathrm{MBq})$. A control group was established with patients who preferred to delay treatment.

Body composition (lean mass, fat mass and bone mineral content) was determined by dualenergy X-ray absorptiometry (DEXA) at baseline and 12 months after.

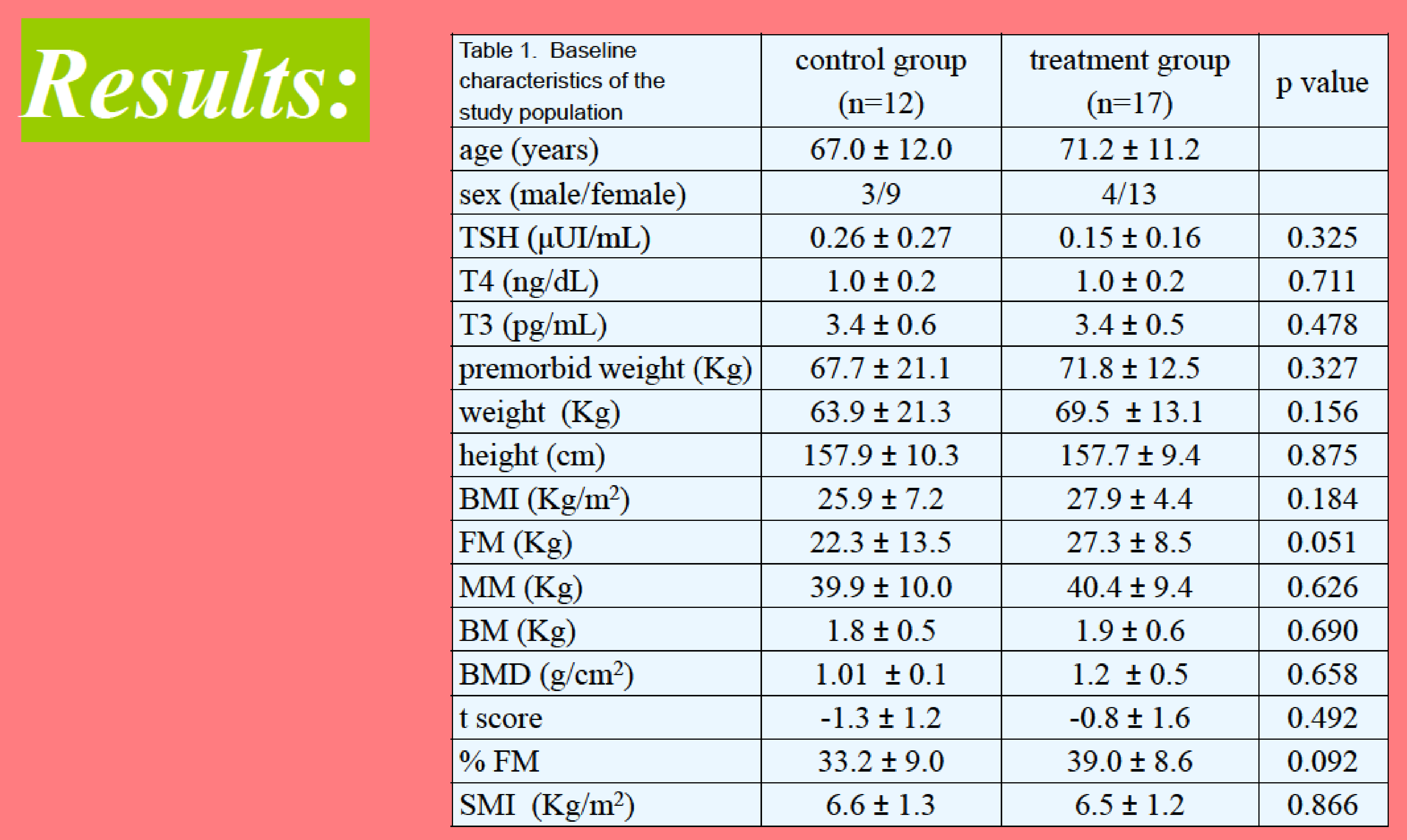

\begin{tabular}{|c|c|c|c|c|c|c|}
\hline $\begin{array}{l}\text { Table 2. } \\
\text { Longitudinal } \\
\text { changes in body } \\
\text { composition }\end{array}$ & \multicolumn{3}{|c|}{ CONTROL GROUP } & \multicolumn{3}{c|}{ TREATMENT GROUP } \\
\hline & $\begin{array}{c}\text { before } \\
(\mathrm{n}=12)\end{array}$ & after $(\mathrm{n}=12)$ & $\mathrm{p}$ value & $\begin{array}{c}\text { before } \\
(\mathrm{n}=17)\end{array}$ & after $(\mathrm{n}=17)$ & $\mathrm{p}$ value \\
\hline weight $(\mathrm{Kg})$ & $63.9 \pm 21.3$ & $65.4 \pm 20.8$ & 0.272 & $69.5 \pm 13.1$ & $70.2 \pm 10.3$ & 0.227 \\
\hline height $(\mathrm{cm})$ & $157.9 \pm 10.3$ & $156.5 \pm 12.4$ & 0.400 & $157.7 \pm 9.4$ & $157.3 \pm 9.5$ & 0.229 \\
\hline $\mathrm{BMI}\left(\mathrm{Kg} / \mathrm{m}^{2}\right)$ & $25.9 \pm 7.2$ & $26.7 \pm 6.9$ & 0.084 & $27.9 \pm 4.4$ & $28.4 \pm 3.7$ & 0.124 \\
\hline $\mathrm{FM}(\mathrm{Kg})$ & $22.3 \pm 13.5$ & $23.6 \pm 13.2$ & 0.084 & $27.3 \pm 8.5$ & $27.8 \pm 6.8$ & 0.093 \\
\hline $\mathrm{MM}(\mathrm{Kg})$ & $39.9 \pm 10.0$ & $40.0 \pm 10.4$ & 0.875 & $40.4 \pm 9.4$ & $40.5 \pm 7.8$ & 0.407 \\
\hline $\mathrm{BM}(\mathrm{Kg})$ & $1.8 \pm 0.5$ & $1.8 \pm 0.5$ & 0.136 & $1.9 \pm 0.6$ & $1.9 \pm 0.5$ & 0.943 \\
\hline $\mathrm{BMD}\left(\mathrm{g} / \mathrm{cm}^{2}\right)$ & $1.0 \pm 0.1$ & $0.8 \pm 0.7$ & 0.937 & $1.2 \pm 0.5$ & $1.0 \pm 0.2$ & 0.679 \\
\hline $\mathrm{t} \mathrm{score}$ & $-1.3 \pm 1.2$ & $-1.3 \pm 1.1$ & 0.722 & $-0.8 \pm 1.6$ & $-0.8 \pm 1.3$ & 0.682 \\
\hline$\% \mathrm{FM}$ & $33.2 \pm 9.0$ & $34.7 \pm 8.9$ & 0.136 & $39.0 \pm 8.6$ & $39.5 \pm 7.2$ & 0.309 \\
\hline $\mathrm{SMI}\left(\mathrm{Kg} / \mathrm{m}^{2}\right)$ & $6.6 \pm 1.3$ & $6.5 \pm 1.4$ & 0.929 & $6.5 \pm 1.2$ & $6.5 \pm 0.9$ & 0.619 \\
\hline & & & & & & \\
\hline
\end{tabular}
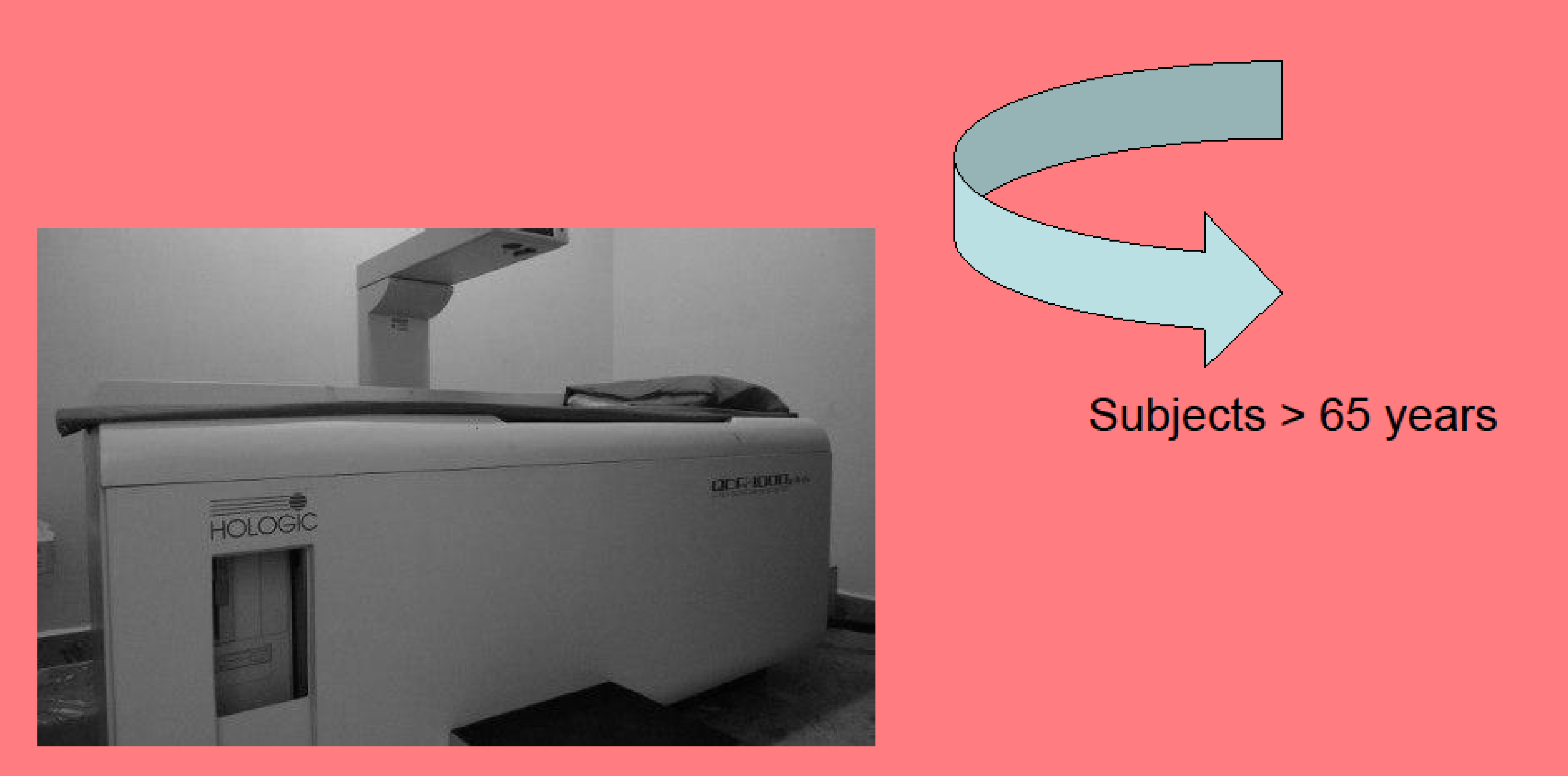

\begin{tabular}{|c|c|c|c|c|c|c|}
\hline \begin{tabular}{|} 
Table 3. \\
Longitudinal \\
changes in body \\
composition in \\
older individuals
\end{tabular} & \multicolumn{3}{|c|}{ CONTROL GROUP } & \multicolumn{3}{c|}{ TREATMENT GROUP } \\
\hline & before (n=7) & after (n=7) & p value & before (n=11) & after (n=11) & p value \\
\hline weight $(\mathrm{Kg})$ & $60.2 \pm 13.8$ & $62.3 \pm 14.4$ & 0.310 & $\mathbf{6 4 . 1} \pm \mathbf{1 0 . 0}$ & $\mathbf{6 6 . 9} \pm \mathbf{9 . 2}$ & $\mathbf{0 . 0 1 0 ^ { * }}$ \\
\hline height $(\mathrm{cm})$ & $158.5 \pm 12.8$ & $157.8 \pm 16.9$ & 0.854 & $153.8 \pm 7.9$ & $153.0 \pm 7.1$ & 0.124 \\
\hline $\mathrm{BMI}\left(\mathrm{Kg} / \mathrm{m}^{2}\right)$ & $24.7 \pm 4.5$ & $25.4 \pm 3.2$ & 0.398 & $27.3 \pm 4.8$ & $28.7 \pm 4.5$ & $0.004^{*}$ \\
\hline $\mathrm{FM}(\mathrm{Kg})$ & $18.0 \pm 0.7$ & $19.5 \pm 0.6$ & 0.237 & $\mathbf{2 6 . 1} \pm \mathbf{8 . 5}$ & $\mathbf{2 7 . 8 \pm 7 . 9}$ & $\mathbf{0 . 0 1 3 ^ { * }}$ \\
\hline $\mathrm{MM}(\mathrm{Kg})$ & $40.4 \pm 10.1$ & $41.0 \pm 11.1$ & 0.398 & $\mathbf{3 6 . 3} \pm \mathbf{0 . 4}$ & $\mathbf{3 7 . 4 \pm 0 . 4}$ & $\mathbf{0 . 0 3 3 ^ { * }}$ \\
\hline $\mathrm{BM}(\mathrm{Kg})$ & $1.7 \pm 0.6$ & $1.8 \pm 0.6$ & 0.128 & $1.6 \pm 0.5$ & $1.8 \pm 0.4$ & 0.328 \\
\hline $\mathrm{BMD}\left(\mathrm{g} / \mathrm{cm}^{2}\right)$ & $1.0 \pm 0.1$ & $0.7 \pm 0.9$ & 0.735 & $1.2 \pm 0.6$ & $1.0 \pm 0.1$ & 0.540 \\
\hline $\mathrm{t} \mathrm{score}$ & $-1.5 \pm 1.4$ & $-1.4 \pm 1.4$ & 0.396 & $-1.0 \pm 1.4$ & $-0.9 \pm 1.3$ & 0.672 \\
\hline$\% \mathrm{FM}$ & $29.8 \pm 8.0$ & $31.4 \pm 7.6$ & 0.499 & $40.1 \pm 8.1$ & $41.0 \pm 7.0$ & 0.248 \\
\hline $\mathrm{SMI}\left(\mathrm{Kg} / \mathrm{m}^{2}\right)$ & $6.6 \pm 1.2$ & $6.8 \pm 1.1$ & 0.600 & $6.0 \pm 0.6$ & $6.3 \pm 0.6$ & $0.033^{*}$ \\
\hline
\end{tabular}

\section{Conclusions:}

References:
Treatment of SHT seems to have positive effects on body composition in subjects older than 65 years. Weight gain reflects increases in fat and lean mass.

1. Cooper DS, Biondi B. Subclinical thyroid disease. Lancet. 2012;379:1142-54

2. Greenlund LJ, Nair KS, Brennan MD. Changes in body composition in women following treatment of overt and subclinical hyperthyroidism Endocr Pract. 2008;14: 973-8. 